\title{
Status of the BSM scenarios
}

\section{Stefan Pokorski*}

University of Warsaw, Faculty of Physics

E-mail: pokorskiefuw.edu.pl

\begin{abstract}
The two main messages from the LHC, after its first phase, are the discovery of the Higgs-like particle and no evidence for any BSM physics. This stunning, continuous, success of the SM up to the mass scales of order $0(1 \mathrm{TeV})$ is very puzzling. Although with the discovery of the Higgs particle, the SM is a consistent theory that can be extrapolated up to the Planck scale, it leaves unanswered several well known experimental and theoretical questions. In particular, the naturalness of the weak scale as the guiding principle for BSM physics is now somewhat challanged. From the historical perspective, the concept of naturalness in particle physics is a crucial issue and it should not be abandoned too quickly. After the lessons from the LHC, supersymmetry still remains to be the leading candidate for BSM physics. Other BSM scenarios and the near term experimental prospects for discovering supersymmetric or non-supersymmetric BSM physics will also be briefly reviewed.
\end{abstract}

Frontiers of Fundamental Physics 14 - FFP14,

15-18 July 2014

Aix Marseille University (AMU) Saint-Charles Campus, Marseille

${ }^{*}$ Speaker. 